\title{
FRAGMENTS OF DYNAMIC OF MÖEBIOUS MAPPINGS AND SOME APPLICATIONS. PART II
}

\author{
Ž. PAVIĆEVIĆ ${ }^{1,2}$, J. ŠUŠIĆ ${ }^{1}$ and M. MARKOVIĆ ${ }^{* 1}$ \\ ${ }^{1}$ Faculty of Natural Sciences and Mathematics, University of Montenegro, \\ Podgorica, Montenegro; \\ ${ }^{2}$ National Research Nuclear University MEPhI \\ (Moscow Engineering Physics Institute), Moscow, Russia \\ *Corresponding author. E-mail: marijanmmarkovic@gmail.com
}

\section{DOI: 10.20948/mathmontis-2020-49-1}

Summary. Using dynamic and geometry of Möebious mappings we prove Lindelöf type theorems for much larger class of functions on the unit disk than previously considered class of meromor-phic functions.

\section{INTRODUCTION}

In classical theory of boundary behaviour of functions of one complex variable and in the theory of boundary sets the special important place is for the Lindelöf theorem and the Fatou theorem (we refer to $[3,12]$ ) on radial and nontangential boundary values of holomorphicc functions. The first one concerns the local property of functions, i.e., it is about the existence of nontanegntial boundary value in a single point in the domain of a holomorphic function, the second one is about global boundary behaviour, i.e., it concerns the almost everywhere existence of radial boundary values of a holomorphic function. Nowadays there exist many proofs of these theorems but all of them use classical results of analytic theory of functions (see [3, 12, 13, 23]). Generalizations of Lindelöf theorems and Fatou theorems goes in many directions. One direction is for analytic functions by proving „stronger“ results, i.e., by proving the existence of nontangentail boundary values under weaker conditions then those in the Lindelof theorem (see [1719]). The second direction is to consider similar theorems for broader class of functions: meromorphic functions, endomorphic mappings, holomorphic mappings of several complex variables, quasiconformal mappings in $n, R n \geq 2$, harmonic functions and similar [22, 24, 25].

In this paper we prove how one can efficasely use the geometry or dynamic of Möebious mappings in order to derive the results on asymptotical behavior of holomorphic functions. Namely, we prove theorems that give necessary and sufficient conditions and criteria in order that a meromorphic function on the unit disk has tangential and nontangential boundary values.

2010 Mathematics Subject Classification: Primary: 30D40, 30D45, 37F45; Secondary: 30C25, 37Fxx.

Keywords and Phrases: Geometric and dynamic of Möebious mappings; normal families of functions; The Lindelöf theorem. 
These theorems show that the conditions in the classical Lindelöf theorem and in the theorem of Lehto and Virtanen, Bagemihl and Seidel, Gavrilov and Burkova on angular boundary values of meromorphic functions may be relaxed. In the proofs of these theorems we use the Main Lemma 1 and the Main Lemma 2 in the Section 5 (see [18]). These results give the necessary and sufficient condition on a function defined on the unit disk in the complex plane, to has a boundary set consisted of one point, along the set which is obtain applying cyclic semi-group produced by an element in the hyperbolic or parabolic Moebius group on the unit disk. More on the topic on boundary asymptotic properties of functions one may found in [13, 17-19, 22-24].

\section{PRELIMINARY NOTATIONS, DEFINITIONS AND RESULTS}

By $D$ we denote the open unit disk $\{z|| z \mid<1\}$ in the complex plane $C$, and with $\Gamma$ we denote the boundary of $D$, and $D^{+}=D \cap\{z \mid \operatorname{Im} z>0\}, \quad D^{-}=D \cap\{z \mid \operatorname{Im} z<0\}$, and $D_{r}=\{z|| z \mid<r\}, 0<\mathrm{r}<1$ is the disk with radius $\mathrm{r}$. By $P_{\theta}$ i $p_{\theta}$ we denote the diameter and the radius of $D$ with one endpoint in $e^{i \theta}$. Further, we denote by $d\left(z_{1}, z_{2}\right)=\left|z_{1}-z_{2}\right|, z_{1}, z_{2} \in \mathbb{C}$ the Euclidean distance on $\mathbb{C}, d_{p h}(z, w)=\left|\frac{z-w}{1-z \bar{w}}\right|$ and $d_{h}(z, w)=\frac{1}{2} \log \frac{1+d_{p h}(z, w)}{1-d_{p h}(z, w)}, z, w \in D$, stand for the pseudohyperbolic and hyperbolic distance between $\mathrm{z}$ and $\mathrm{w}$ in the dsik $\mathrm{D}$, respecitvely, and

$d_{s}(z, w)=\left\{\begin{array}{l}\frac{2|z-w|}{\sqrt{1+|z|} \cdot \sqrt{1+|w|}}, \mathrm{z}, w \in C ; \\ \frac{1}{\sqrt{1+|z|^{2}}}, \mathrm{z} \in C, \mathrm{w}=\infty\end{array}\right.$

is the spherical distance on the Rimanian sphere $\overline{\mathbb{C}}=\mathbb{C} \cup\{\infty\}$.

It is well known that $d_{h}$ is the metric in the Poencare model of the hyperbolic geometry on the disk $D$ introduced by Lobachevsy.

All convergencies in this paper are with respect to the distances introduces above.

The set $D_{p h}\left(w, r^{\prime}\right)=\left\{z \mid z \in D, d_{p h}(z, w)<r^{\prime}\right\}, w \in D, 0<r^{\prime}<1$, is the pseudohyperbolic disk, and $D_{h}(w, r)=\left\{z \mid z \in D, d_{h}(z, w)<r\right\}, w \in D, r>0$, is the disk with respect to the hyperbolic distance.

Lemma 1. We have $D_{h}(w, r)=D_{p h}\left(w, r^{\prime}\right)$, where $r=\frac{1}{2} \ln \frac{1+r^{\prime}}{1-r^{\prime}}\left(r^{\prime}=\frac{e^{2 r}-1}{e^{2 r}+1}=t h r\right)$. 
The pseudohyperbolic disk $D_{p h}(w, r)$ is the Euclidean disk $D(c, R)=\{z \in D|| z-c \mid<R\}$ for $c=\frac{1-r^{2}}{1-r^{2}|w|^{2}}$, and $R=\frac{1-|w|^{2}}{1-r^{2}|w|^{2}} r$.

Therefore, the boundaries of hyperbolic and pseudohyperbolic disks are the ordinary cycles. The cycle which lies in $D$ and with $\Gamma$ has one common point is the oricycle $D$. The radius of $D$ and arcs in $D$ and in intersection with $\Gamma$ have two points are hypercycles in $D$.

An arbitrary hypercycle will be denoted by $\mathrm{H}$, an arbitrary oricycle will be denoted by $\mathrm{O}$. We denote by $H^{\theta}, \theta \in[0, \pi)$, the hypercycle which connects the points $-e^{i \theta}$ and $e^{i \theta}$; we denote by $O^{\theta}, \theta \in[0,2 \pi)$, the oricycle which is tangent to $\Gamma$ in $e^{i \theta}$, and $O_{0}^{\theta}=\left\{\frac{u}{u-i} e^{i \theta} \mid u \in(-\infty, \infty)\right\}$ is the oricycle $\left\{z|| z-\frac{1}{2} e^{i \theta} \mid=\frac{1}{2}\right\}$.

We will also consider the family of all hypercycles with two common points in $\Gamma$.

The hyperbolic distance between a point $\mathrm{z}, \mathrm{z} \in D$, to the curve $\gamma, \gamma \subset D$, is $d_{h}(z, \gamma)=\inf _{w \in \gamma} d_{h}(z, w)$.

For $\gamma=H^{\theta}$, one can prove that $d_{h}\left(z, H^{\theta}\right)=\min _{w \in H^{\theta}} d_{h}(z, w)$ and that $d_{h}\left(z, H^{\theta}\right)$ does not depend on $\mathrm{z}$ if $\mathrm{z} \in \mathrm{H}$, where $\mathrm{H}$ is a hyper-cycle from the family of all hypercycles which is defined by the hypercycle $H^{\theta}$ (see [10]). Also one can prove (see [10]) that there exists unique point $w_{0}$ in $H^{\theta}$ such that

$$
d_{h}\left(z, H^{\theta}\right)=\min _{w \in H^{\theta}} d_{h}(z, w)=d_{h}\left(z, w_{0}\right) .
$$

From above, by "symmetric thinking”, it follows that for $w \in H^{\theta}$ there exists unique point $z_{0}$ in $H$ such that

$$
d_{h}(w, H)=\min _{z \in H} d_{h}(w, z)=d_{h}\left(w, z_{0}\right)
$$

And this distance does not depend on $w \in H^{\theta}$.

From (1) and (2) it follows that for any $w \in H^{\theta}$ and $z \in H$ there exists unique points $w_{0} \in H^{\theta}$ and $z_{0} \in H$ such that

$$
d_{h}(w, H)=d_{h}\left(z, H^{\theta}\right)=d_{h}\left(w_{0}, z_{0}\right) .
$$


Having in mind all the preceding, the equality (3) define the hyperbolic distance between hypercycles $H^{\theta}$ and $H$. Notation: $d_{h}\left(H^{\theta}, H\right)$.

From the all given above we have:

Lemma 2 (see [10]). The set of points in D such the hyperbolic distance between the hypercycle $H$ is the hypercycle which belongs to the family of all hypercycles defined by the hypercycle $H$.

From Lemma 2 we obtain:

Lemma 3 (see $[8,14])$. The set $\Delta_{H}(\theta, r)=\bigcup_{a \in(-1,1)} D_{h}\left(a e^{i \theta}, r\right), r \in(0,+\infty)$, is a domain in the disk $D$ bounded by two hyper-cycles $H^{\theta}(r)$ and $H^{\theta}(-r)$ such that their hyperbolic distance to the radius $P_{\theta}$ is equal to $r$, and which contains the points - th $r e^{i\left(\frac{\pi}{2}+\theta\right)}$ and th $r e^{i\left(\frac{\pi}{2}+\theta\right)}$ and contains the points $-e^{i \theta}$ and $e^{i \theta}$ (see the Figure 1).

Lemma 4 (see [10]). Let $r$ be the hyperbolic distance of hyper-cycle $H^{\theta}$ from the diameter $P_{\theta}$ of the disk $D$. The angle $\alpha$ between $H^{\theta}$ and $P_{\theta}$ is equal to $\alpha=\frac{\pi}{2}-\operatorname{arctg} e^{ \pm 2 r \sqrt{\pi}}$.

If $h\left(\theta, \alpha_{1}\right)$ and $h\left(\theta, \alpha_{2}\right),-\frac{\pi}{2}<\alpha_{1}<\alpha_{2}<\frac{\pi}{2}$, are $\operatorname{arcs}$ in $D$ that with the radius $p_{\theta}$ of the disk $D$ with endpoint in point $e^{i \theta}$ make angles $\alpha_{1}$ and $\alpha_{2}$, then the domain in $D$ which is bounded by these arcs and by the circle $D_{r}=\left\{z|| z-e^{i \theta} \mid=r\right\}$ is the Stolz angle with vertex at $e^{i \theta}$. This domain is denoted by $\Delta\left(\theta, \alpha_{1}, \alpha_{2}\right)$. By $\Delta(\theta, \alpha)$ we denote the Stolz angle with boundary $h(\theta, \alpha)$ and $h(\theta,-\alpha),-\frac{\pi}{2}<\alpha<\frac{\pi}{2}$. We denote it by $\Delta\left(\theta, \alpha_{1}, \alpha_{2}\right)$. With $\Delta(\theta, \alpha)$ we denote the Stolz angle with boundary $h(\theta, \alpha)$ and $h(\theta,-\alpha),-\frac{\pi}{2}<\alpha<\frac{\pi}{2}$, i.e., $\Delta(\theta, \alpha)=\left\{z|\mathrm{z} \in \mathrm{D},| \arg \left(\mathrm{e}^{\mathrm{i} \theta}-\mathrm{z}\right) \mid<\alpha, 0<\alpha<\frac{\pi}{2}\right\}$.

Threfore, the Stolz angle is the domain which is an usualty geometic object (see Figure 1).

From Lemma 4 we obtain:

Lemma 5. For every $\alpha,-\frac{\pi}{2}<\alpha<\frac{\pi}{2}$, there exist $r, r \in(0,+\infty), r_{1}, r_{1} \in(0,1)$, such that $\left\{z|| y-e^{i \theta} \mid<r_{1}\right\} \cap \Delta(\theta, \alpha) \subset\left\{z|| y-e^{i \theta} \mid<r_{1}\right\} \cap \Delta_{H}(\theta, r)$. For every $r, r \in(0,+\infty)$, there exists $\alpha,-\frac{\pi}{2}<\alpha<\frac{\pi}{2}$, such that $\Delta_{H}(\theta, r) \subset \Delta(\theta, \alpha)$. 


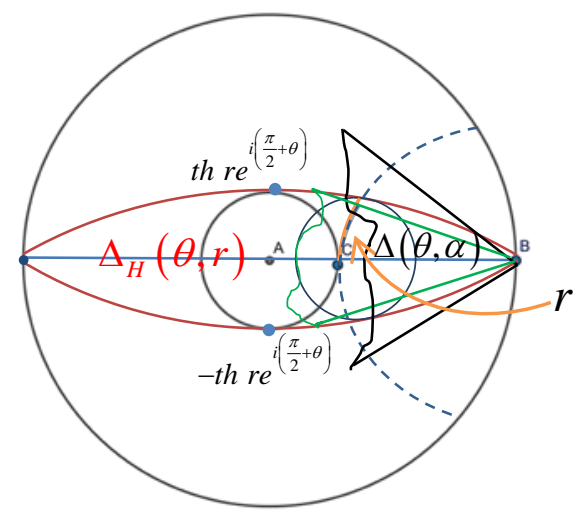

Figure 1

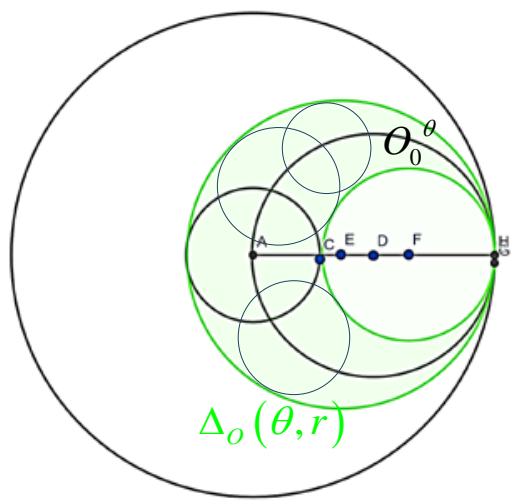

Figure 2

If $d_{h}(z, \gamma)=\inf _{w \in \gamma} d_{h}(z, w)$ and if $\gamma=O^{\theta}$, then $d_{h}\left(z, O^{\theta}\right)=\min _{w \in O^{\theta}} d_{h}(z, w)$ and $d_{h}\left(z, H^{\theta}\right)$ do not depend on $z \in O$, where $O$ is the oricycle from the family of all oricycles generated by $O^{\theta}$. One can also prove that (see[10]) there exists only one point $w_{0}$ in $O^{\theta}$ such that $d_{h}\left(z, O^{\theta}\right)=\min _{w \in O^{\theta}} d_{h}(z, w)=d_{h}\left(z, w_{0}\right)$. Analogy one may define the distance between two oricycles from the same family of ori-cycles in the following way $d_{h}\left(H^{\theta}, H\right)=d_{h}(w, H)=d_{h}\left(z, H^{\theta}\right)$. It may be shown that there exist unique points $w_{0} \in O^{\theta}$ and $z_{0} \in O$ such that $d_{h}\left(H^{\theta}, H\right)=d_{h}(w, H)=d_{h}\left(z, H^{\theta}\right)=d_{h}\left(w_{0}, z_{0}\right)$.

Now, we have the following statements:

Lemma 6 (see [10]). The set of points in D for which the hyperbolic distance is constant from the oricycle $O$ is the orycycle in the family of all orycycles defined by the oricycle $O$.

From lemma 6, we have:

Lemma 7 (see [14]). The set $\Delta_{O}(\theta, r)=\bigcup_{u \in(-\infty, \infty)} D_{h}\left(\frac{u}{u+i} e^{i \theta}, r\right), r \in(0,+\infty)$, is a domain in the disk $D$ which is bounded by two ori-cycles $O^{\theta}(-r)$ and $O^{\theta}(r)$ such that the hyperbolic distance between them and the oricycle $O_{0}{ }^{\theta}=\left\{\frac{u}{u+i} e^{i \theta} \mid u \in(-\infty, \infty)\right\}$ is equal to $r$, and that pass throughout $e^{i \theta}$, -th $r e^{i \theta}$ and th $r e^{i \theta}$ (see Figure 2). 


\section{FRAGMENTS OF THE GEOMETRY OF MÖBIOUS MAPPINGS}

The Möbious group on the unit disk D is the group of all conformal automorphisms of the unit disc D, i.e., $G=G(D)=\left\{e^{i \theta} \frac{z-a}{1-\bar{a} z} \mid a \in D, z \in \mathbb{C}, \theta \in[0,2 \pi)\right\}$.

The set

$$
H_{D}^{\theta}=\left\{g_{a}^{\theta}=g_{a}^{\theta}(z)=\frac{z+a e^{i \theta}}{1+a e^{-i \theta} z} \mid a \in(-1,1)\right\}, \theta \in[0, \pi) \text { is fixed, }
$$

stand for the hyperbolic subgroup of $G$ with fixed points $e^{i \theta}$ and $-e^{i \theta}$,

$$
P_{D}^{\theta}=\left\{g_{u}^{\theta}=g_{u}^{\theta}(z)=\frac{(u+i) z-u e^{i \theta}}{-(u-i)+u e^{-i \theta} z} \mid u \in(-\infty,+\infty)\right\}, \theta \in[0,2 \pi) \text { is fixed, }
$$

is the parabolic subgroup of $G$ with fixed point $e^{i \theta}$,

and finally

$$
E_{D}^{\theta}=\left\{g_{\theta}^{z_{0}}=g_{\theta}^{z_{0}}(z)=\frac{\left(1-\left|z_{0}\right| e^{i \theta}\right) z-z_{0}\left(1-e^{i \theta}\right)}{\overline{z_{0}}\left(1-e^{i \theta}\right) z+e^{-i \theta}-\left|z_{0}\right|^{2}} \mid \theta \in[0,2 \pi)\right\}, z_{0} \in D \text { is fixed, }
$$

is the elliptic subgroup of $G$ with fixed point $z_{0}$.

Since the hyperbolic distance is invariant with respect to $g \in G$ and from the definition of the groups $H_{D}^{\theta}$ and $P_{D}^{\theta}$ and sets $P_{D}^{\theta}, \Delta_{H}(\theta, r), O_{0}{ }^{\theta}$ and $\Delta_{O}(\theta, r)$ we have the following statements:

Lemma 9. (i) $\Delta_{H}(\theta, r)=\underset{g \in H_{D}^{\theta}}{\cup} g\left(D_{h}(0, r)\right)=\underset{g \in H_{D}^{\theta}}{\cup} g\left(D_{p h}(0, t h r)\right), r \in(0,+\infty)$

(ii) $\Delta_{P}(\theta, r)=\underset{g \in P_{D}^{\theta}}{\cup} g\left(D_{h}(0, r)\right)=\underset{g \in P_{D}^{\theta}}{\cup} g\left(D_{p h}(0, t h r)\right), r \in(0,+\infty)$.

The set $A, A \subset D$, is the stabilisator of the group $H_{D}^{\theta}$ if $g(A)=A$, for every $g \in H_{D}^{\theta}$.

Lemma 10. For every $g \in H_{D}^{\theta}$ we have $g\left(P_{\theta}\right)=P_{\theta}$, i.e., the diameter $P_{\theta}$ is stabilisator of the group $H_{D}^{\theta}$.

Lemma 11. For every $g \in H_{D}^{\theta}$ and $r \in(0,+\infty)$ we have $g\left(\Delta_{H}(\theta, r)\right)=\Delta_{H}(\theta, r)$, i.e., the set $\Delta_{H}(\theta, r)$ is also the stabilisator of the group $H_{D}^{\theta}$.

Lemma 12. For every $g \in P_{D}^{\theta}$ we have $g\left(O_{0}^{\theta}\right)=O_{0}^{\theta}$, i.e., the ori-cycle $O_{0}^{\theta}$ is the stabilisator of the group $P_{D}^{\theta}$. 
Lemma 13. For every $g \in P_{D}^{\theta}$ and $r \in(0,+\infty)$ we have $g\left(\Delta_{O}(\theta, r)\right)=\Delta_{O}(\theta, r)$, i.e., $\Delta_{O}(\theta, r)$ is the stabilisator of the group $P_{D}^{\theta}$.

For $g \in G(D)$ denote $g^{n}(z)=\underbrace{g(g(\ldots(g)}_{n \text { puta }}(z) \ldots)), \quad g^{0}(z)=i, \quad i$ is the identity and

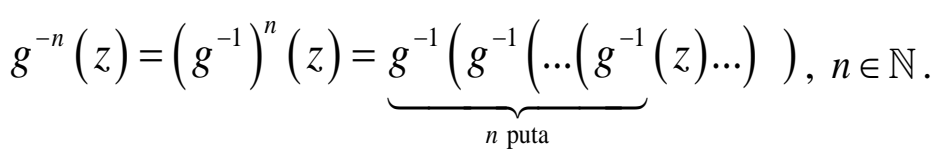

Lemma 14 (see [2 ] on p. 73).

(i) Let $g \in H_{D}^{\theta}$. For fixed points $e^{i \theta}$ and $-e^{i \theta}$ there holds $g^{n}(z) \underset{n \rightarrow \infty}{\rightarrow} e^{i \theta}$ and $g^{-n}(z) \underset{n \rightarrow \infty}{\rightarrow}-e^{i \theta}$, where we mean uniform convergence on compacts sets of the disk $D$.

(ii) Let $g \in P_{D}^{\theta}$. Then for fixed point $e^{i \theta}$ we have $g^{n}(z) \underset{n \rightarrow \infty}{\rightarrow} e^{i \theta}$, where we also mean the uniform convergence of compact subsets of the disk $D$.

Therefore, the point $e^{i \theta}$ is an attraction point for $g \in H_{D}^{\theta}$, and $-e^{i \theta}$ is repulsive point for g, i.e. it is an attraction point for $g^{-1}$. If $g \in P_{D}^{\theta}$ then the attraction point for $g \in P_{D}^{\theta}$.

For $g \in H_{D}^{\theta}$, and $\theta \in[0, \pi)$ fixed, $g \neq i$, denote $H_{g}^{\theta}=\left\{g^{n} \mid n \in \mathbb{Z}\right\}$. The set $H_{g}^{\theta}$ sa with composition operation is the cyclic subgroup of the group $H_{D}^{\theta}$. If $g \in P_{D}^{\theta}$, then the set $P_{g}^{\theta}=\left\{g^{n} \mid n \in \mathbb{Z}\right\}$ with composition of functions is the cyclic subgroup of $P_{D}^{\theta}$.

Let $\Delta_{g}(\theta, r)=\cup_{n \in \mathbb{Z}} g^{n}\left(D_{h}(0, r)\right), r \in(0,+\infty)$.

Further, from the property of invariance of the hyperbolic distance with respect to $g \in G$ we have:

Lemma15. $\Delta_{g}(\theta, r)=\cup_{n \in \mathbb{Z}}\left(D_{h}\left(g^{n}(0), r\right)\right), r \in(0,+\infty)$.

Lemma16. Let $g \in H_{D}^{\theta}, g \neq i$. For every $r \in(0,+\infty)$ there exists $r_{1} \in(0,+\infty)$ such that $\Delta_{H}(\theta, r) \subset \Delta_{g}\left(\theta, r_{1}\right)$, and $\Delta_{g}(\theta, r) \subset \Delta_{H}(\theta, r)$ for every $r \in(0,+\infty)$.

Proof of lemma 16. Let $g \in H_{D}^{\theta}$ be arbitrary and let it be fixed and $g \neq i$. Let $z \in \Delta_{H}(\theta, r)$. There exists $a \in(0,+\infty)$ such that $z \in D_{h}\left(a e^{i \theta}, r\right)$. Since $a e^{i \theta} \in P_{\theta}$ and $g^{n}(0) \in P_{\theta}$ for every $n \in \mathbb{Z}$, there exists $N \in \mathbb{Z}$ such that $a e^{i \theta}$ is between $g^{N}(0)$ and $g^{N+1}(0)$ or is equalt to one of that points. Let $0<M=d_{h}(0, g(0))=d_{h}\left(g^{n}(0), g^{n+1}(0)\right), n \in \mathbb{Z}$. Then we have 
$d_{h}\left(g^{N}(0), z\right) \leq d_{h}\left(g^{N}(0), a e^{i \theta}\right)+d_{h}\left(a e^{i \theta}, z\right) \leq d_{h}\left(g^{N}(0), g^{N+1}(0)\right)+d_{h}\left(a e^{i \theta}, z\right)<M+r$

Therefore, for every $z \in \Delta_{H}(\theta, r)$ there exists $N \in \mathbb{Z}$ such that $d_{h}\left(g^{N}(0), z\right)<M+r$, where $\mathrm{M}$ and $\mathrm{r}$ are independent on $\mathrm{z}$ and $\mathrm{N}$.

Since $\quad \Delta_{g}(\theta, M+r)=\underset{n \in \mathbb{Z}}{\cup}\left(D_{h}\left(g^{n}(0), M+r\right)\right) \quad$ (by Lemma 14) and $D_{h}\left(g^{N}(0), M+r\right) \subset \Delta_{g}(\theta, M+r)$, we obtain $z \in \Delta_{g}(\theta, M+r)$. If we take $r_{1}=M+r$, it follows $\Delta_{H}(\theta, r) \subset \Delta_{g}\left(\theta, r_{1}\right)$.

Now $\Delta_{g}(\theta, r) \subset \Delta_{H}(\theta, r), r \in(0,+\infty)$ follows from Lemma 9 and Lemma 15 . $\square$

Lemma 17. Let $g \in P_{D}^{\theta}, g \neq i$. For every $r \in(0,+\infty)$ there exists $r_{1} \in(0,+\infty)$ such that $\Delta_{P}(\theta, r) \subset \Delta_{g}\left(\theta, r_{1}\right)$, and $\Delta_{g}(\theta, r) \subset \Delta_{P}(\theta, r)$ for every $r \in(0,+\infty)$.

Lemma 17 may be proved in a similar way as Lemma 16, instead of diameter $P_{\theta}$ one has to take the oricycle $O_{0}^{\theta}$.

We will further consider the domains: $\tilde{\Delta}_{H}(\theta, r)=\bigcup_{a \in[0,1]} D_{h}\left(a e^{i \theta}, r\right) \quad$ and $\tilde{\tilde{\Delta}}_{H}(\theta, r)=\bigcup_{a \in[-1,0]} D_{h}\left(a e^{i \theta}, r\right), \quad r \in(0,+\infty)$, we call them the hypercyclic domains in $\mathrm{D}$ and $\tilde{\Delta}_{o}(\theta, r)=\bigcup_{u \in(0,+\infty)} D_{h}\left(\frac{u}{u+i} e^{i \theta}, r\right)$ and $\tilde{\tilde{\Delta}}_{o}(\theta, r)=\bigcup_{u \in(-\infty 0,)} D_{h}\left(\frac{u}{u+i} e^{i \theta}, r\right), r \in(0,+\infty)$, which will be called the oricyclic domains in $\mathrm{D}$.

Lemma 18. Let $g_{a} \in H_{D}^{\theta}, g_{a} \neq i$, for which $e^{i \theta}$ is an attraction fixed point. Then for every $r \in(0,+\infty)$ there exists $r_{1} \in(0,+\infty)$ such that $\bigcup_{n=0}^{\infty} g_{a}^{n}\left(D_{h}(0, r)\right) \subset \tilde{\Delta}_{H}(\theta, r) \subset \bigcup_{n=0}^{\infty} g_{a}^{n}\left(D_{h}\left(0, r_{1}\right)\right)$.

Lemma 19. $\operatorname{Let}_{u} \in P_{D}^{\theta}, g_{u} \neq i$, for which $e^{i \theta}$ is fixed attraction point. Then for every $r \in(0,+\infty)$ there exists $r_{1} \in(0,+\infty)$ such that $\bigcup_{n=0}^{\infty} g_{u}^{n}\left(D_{h}(0, r)\right) \subset \tilde{\Delta}_{o}(\theta, r) \subset \bigcup_{n=0}^{\infty} g_{u}^{n}\left(D_{h}\left(0, r_{1}\right)\right)$, $u>0$, and $\bigcup_{n=0}^{\infty} g_{u}^{n}\left(D_{h}(0, r)\right) \subset \tilde{\tilde{\Delta}}_{o}(\theta, r) \subset \bigcup_{n=0}^{\infty} g_{u}^{n}\left(D_{h}\left(0, r_{1}\right)\right), u<0$.

Lemma 18 and lemma 19 may be proved in a similar way as Lemma 16. 


\section{CLASSICAL RESULTS FOR ASYMPTOTIC AND ANGULAR LIMIT VALUES OF ANALYTIC FUNCTIONS AT A POINT}

For $A \subset D, \overline{\mathrm{A}} \cap \Gamma=\left\{e^{i \theta}\right\}$, we denote by $\bar{A}$ the closure of the set $\mathrm{A}$, and $C\left(f, A, e^{i \theta}\right)=\left\{\omega \mid \omega \in \Omega,\left(\mathrm{z}_{\mathrm{n}}\right) \subset A, \lim _{\mathrm{n} \rightarrow \infty} z_{n}=e^{i \theta}, \lim _{n \rightarrow \infty} f\left(z_{n}\right)=\omega\right\}$ is the boundaty set of a function $f: D \rightarrow \Omega$ corresponding to the point $e^{i \theta}$ allong the set $A$. It is known that $C\left(f, A, e^{i \theta}\right)=\overline{C\left(f, A, e^{i \theta}\right)}$.

The symbol $\varphi_{\mathrm{n}} \rightrightarrows_{\mathrm{K}} \varphi$ denotes the uniform convergence on the set $K \subset D$, of the sequence $\left(\varphi_{n}\right)$ of functions $\varphi_{n}: D \rightarrow \overline{\mathbb{C}}, n \in \mathbb{N}$, to $\varphi: D \rightarrow \overline{\mathbb{C}}$.

If $A=\Delta\left(e^{i \theta}, \alpha\right)$ is a Stolz angle in the disk $D$ with the vertex at the point $e^{i \theta}$, then $C\left(f, \Delta\left(e^{i \theta}, \alpha\right), e^{i \theta}\right)$ is the boundary set of the function $f$ along the angle $\Delta\left(e^{i \theta}, \alpha\right)$. If for every $\alpha, 0<\alpha<\frac{\pi}{2}, C\left(f, \Delta\left(e^{i \theta}, \alpha\right), e^{i \theta}\right)=\{\omega\}$, the $e^{i \theta}$ is the Fatou point of $\mathrm{f}$, and $\omega \in \Omega$ is the unique nontangential boundary value.

We always denote by $\gamma$ the simple Jordan curve in the disk $D$ with endpoint in $e^{i \theta}$. If $C\left(f, \gamma, e^{i \theta}\right)=\{\omega\}, \omega \in \Omega$, then $\omega$ is an asympthotic boundary value of the function $\mathrm{f}$ in the point $e^{i \theta}$ along the curve $\gamma$.

We give now the classical assymptotic results and nontangential of analytic functions.

Theorem of Lindelöf (see [12, 23]). If $f: D \rightarrow \mathbb{C}$ is a bounded analytic function. If $C\left(f, \gamma, e^{i \theta}\right)=\{\omega\}, \omega \in \mathbb{C}$, then $C\left(f, \Delta\left(e^{i \theta}, \alpha\right), e^{i \theta}\right)=\{\omega\}$, i.e., $e^{i \theta}$ is the Fatou point of function $f$.

There are many proofs of the Lindelöf theorem. A proof based on maximum principle of analytic functions may be found in [23].

One generalization of the Lindelöf theorem is given by Lehto and Virtanen in [11]. The used results from normal function theory and results in harmonic function theory and harmonic measure.

For a family of functions $\mathfrak{I}=\{f \mid f: O \rightarrow \overline{\mathbb{C}}\}$ we say that it is normal family on a domain $O$,

$O \subset \mathbb{C}$, if for every sequence $\left(f_{n}\right)$ in that familty $\mathfrak{I}$ there exists a subsequence $\left(f_{n_{k}}\right)$ which 
convege uniformly on compact subsets of $O$ to a function $f: O \rightarrow \overline{\mathbb{C}}$. This is normality in the sense of $\mathfrak{I}$ of Montel. The family of functions $\mathfrak{I}=\{f \mid f: O \rightarrow \overline{\mathbb{C}}\}$ is normal in the point $z \in O$ if it is normal familiy in a neiborhoud of $z$.

It is well known that a family of functions $\mathfrak{I}=\{f \mid f: O \rightarrow \overline{\mathbb{C}}\}$ is normal family in the domain $O$ if and only if it is normal in every point in the domain $\mathrm{O}$ (see [16, 20]).

If $O \subset \overline{\mathbb{C}}$, i.e. if $\infty \in O$, then the family of functions $\mathfrak{J}=\{f \mid f: O \rightarrow \overline{\mathbb{C}}\}$ is normal in the point $\infty$ if we have normality of the family $\mathfrak{I}^{\prime}=\left\{f\left(\frac{1}{z}\right) \mid f \in \mathfrak{J}\right\}$ in 0 . The family of functions $\mathfrak{I}=\{f \mid f: O \rightarrow \overline{\mathbb{C}}\}$ is normal on $O$ if it is normal in every point of the domain $O$. The theory of normal functions is well exposed in [16, 20].

If $f: D \rightarrow \mathbb{C}$ is a bounded analytic maping, then the family $\{f \circ g \mid g \in G\}$ is normal family of functions on the disk $D$.

Theorem of Lehto and Virtanen (see [11]). Let $f: D \rightarrow \overline{\mathbb{C}}$ be a meromorphic function. If $\{f \circ g \mid g \in G\}$ is normal family of functions on the disk $D$ and $C\left(f, \gamma, e^{i \theta}\right)=\{\omega\}, \omega \in \mathbb{C}$, then we have $C\left(f, \Delta\left(e^{i \theta}, \alpha\right), e^{i \theta}\right)=\{\omega\}$, i.e., $e^{i \theta}$ is the Fatou point of the function $f$.

For the proof of the theroem Lehto and Virtanen used the results from harmonic function theory and harmonic measures (the theorem on two constants) and the propery of the normal meromorphic functions (see $[3,11])$.

A meromorphic function $f: D \rightarrow \overline{\mathbb{C}}$ for which the family $\{f \circ g \mid g \in G\}$ is normal family of functions on $\mathrm{D}$ is the class of very well understood normal meromorphic functions $\mathrm{N}$ which contains the Bloch class of holomorphic functions denoted by B.

In the following theorems proved by Bagemihl and Seidel [1], it is proved the existence of angular boundary values under weaker asymptotical conditions then these in the preceding theorems. But these theorems are based on the theorems of Lehto and Virtanen.

Theorem of Bagemihl and Seidel 1 (see [1]). Let $f: D \rightarrow \overline{\mathbb{C}}$ be a meromorphic function. If $\{f \circ g \mid g \in G\}$ is a normal family of functions on the disk $D$ and if for every $z \in D$ we have $f(z) \neq w, w \in \overline{\mathbb{C}}$, and if there exists a sequence $\left(z_{n}\right), z_{n} \in D, n \in \mathbb{N}$, such that: 
$\lim _{n \rightarrow \infty} z_{n}=e^{i \theta}, d_{h}\left(z_{n}, z_{n+1}\right)<M, n \in \mathbb{N}$, i $\lim _{n \rightarrow \infty} f\left(z_{n}\right)=\omega, \omega \in \overline{\mathbb{C}}$, then $C\left(f, \Delta\left(e^{i \theta}, \alpha\right), e^{i \theta}\right)=\{\omega\}$ for every $\alpha, 0<\alpha<\frac{\pi}{2}$, i.e., $e^{i \theta}$ is the Fatou point of the function $f$.

Theorem of Bagemihl and Seidel 2 (see [1]). Let $f: D \rightarrow \overline{\mathbb{C}}$ be a meromorphic function. If $\{f \circ g \mid g \in G\}$ is a normal family of functions on the disk $D$ and if there exists a sequence $\left(z_{n}\right), z_{n} \in D, n \in \mathbb{N}$, such that $\lim _{n \rightarrow \infty} z_{n}=e^{i \theta}, \lim _{n \rightarrow \infty} d_{h}\left(z_{n}, z_{n+1}\right)=0$, i $\lim _{n \rightarrow \infty} f\left(z_{n}\right)=\omega, \omega \in \overline{\mathbb{C}}$, then $C\left(f, \Delta\left(e^{i \theta}, \alpha\right), e^{i \theta}\right)=\{\omega\}$ for every $\alpha, 0<\alpha<\frac{\pi}{2}$, i.e., $e^{i \theta}$ is the Fatou point of function $f$.

Bagemihl and Seidel [1] constructed an analytic functions in order to show that the condition concerning the hypervbolic distance $d_{h}\left(z_{n}, z_{n+1}\right)$ in Theorem 5 and Theorem 6 is not possible to remove.

In the following theorem proved by Gavrilov and Burkova in [8], it is proved the existence of angular boundary values for the broader class of meromorphic functions then the class in the theorem of Lehto and Virtanen. In [Gavrilov and Burkova 11] it is given an example of meromorphic function for which $\left\{f \circ g_{a}^{\theta} \mid g_{a}^{\theta} \in H_{D}^{\theta}\right\}$ is normal on $D$ but the family $\{f \circ g \mid g \in G\}$ is not normal on $D$.

A construction is based on the theorem which says that for a meromorphic function $f: D \rightarrow \bar{C}$ the family $\{f \circ g \mid g \in G\}$ is normal on the disku $D$ if and only if the disk $D$ does not contain the so called P-sequences for the function f, dok je $\left\{f \circ g_{a}^{\theta} \mid g_{a}^{\theta} \in H_{D}^{\theta}\right\}$ is normal family on the disk $D$ if and only if in the domain $\Delta_{g}(\theta, r) \subset \Delta_{H}(\theta, r), r \in(0,+\infty)$, does not exist the P-sequences for the function $\mathrm{f}$.

A sequence $\left(z_{n}\right), z_{n} \in D, \lim _{n \rightarrow \infty}\left|z_{n}\right|=1$, is a P-sequence for a function $f: D \rightarrow \bar{C}$ if for every subsequence $\left(z_{n_{k}}\right)_{k \in N}$ and for every $\varepsilon, 0<\varepsilon<1$, the function $f$ on $\bigcup_{k \in N} D_{h}\left(z_{n_{k}}, \varepsilon\right)$, takes inifinity many times all velues in $\bar{C}$, except possibly at most two (see definition, Gavrilov[6]). In the sequel we will need the following theorems concerning the $P$-sequences: 
Theorem on P-sequences 1 (see [6, Lemma 1]). Let $\left(z_{n}\right)$ be a P-sequence for a meromorphic function $f: D \rightarrow \bar{C}$. If for a sequence $\left(z_{n}^{\prime}\right) \subset D$ we have $\lim _{n \rightarrow \infty} d_{h}\left(z_{n}, z_{n}^{\prime}\right)=0$, then the sequence $\left(z_{n}^{\prime}\right)$ is P-sequence for $f$.

Theorem on P-sequences 2 (see [26]). Let $f: D \rightarrow \bar{C}$ be a meromorphic fuction on $D$ and let $\left(z_{n}\right) \subset D$ a sequence such that $\lim _{n \rightarrow \infty}\left|z_{n}\right|=1$ and $\lim _{n \rightarrow \infty} f\left(z_{n}\right)=c$ for some a $c \in \overline{\mathbb{C}}$. Further, let $\left(z_{n}^{\prime}\right) \subset D$ be a sequence such that $\lim _{n \rightarrow \infty}\left|z_{n}^{\prime}\right|=1, \lim _{n \rightarrow \infty} d_{h}\left(z_{n}, z_{n}^{\prime}\right)=0$, and $\left(f\left(z_{n}\right)\right)$ does not sonverge to a c as $n \rightarrow \infty$. Then $\left(z_{n}\right)$ and $\left(z_{n}^{\prime}\right)$ are both P-sequences of the fuction $f$.

Theorem of Gavrilov and Burkova (see [8]). Let $f: D \rightarrow \overline{\mathbb{C}}$ be a meromorphic function. If $\left\{f \circ g_{a}^{\theta} \mid g_{a}^{\theta} \in H_{D}^{\theta}\right\}$ is a normal family on the disk $D$ and $C\left(f, \gamma, e^{i \theta}\right)=\{\omega\}, \omega \in \mathbb{C}$, then $C\left(f, \Delta\left(e^{i \theta}, \alpha\right), e^{i \theta}\right)=\{\omega\}$ for every $\alpha, 0<\alpha<\frac{\pi}{2}$, i.e., $e^{i \theta}$ is the Fatou point of the function $f$.

A proof of theorem of Gavrilov and Burkova goes in the same way as the, by using the result from harmonic function theory as well as using properties of harmonic measure, as in the proof of theorem Lehto - Virtanen.

In [27] are given theorems that are analigies to the theorems of Bagemil and Seidel for meromorphic functions on $D$ i.e., functions for which $\left\{f \circ g_{a}^{\theta} \mid g_{a}^{\theta} \in H_{D}^{\theta}\right\}$ is normal on the disk D.

\section{MAIN RESULT}

The main lemma 1. For any function $f: D \rightarrow \overline{\mathbb{C}}$, any compact set $K, K \subset D$, and any mapping $g_{a} \in H_{D}^{\theta}, g_{a} \neq i$, the following conditions are equivalent:

$$
\begin{aligned}
& \text { i) } \quad f_{\circ}\left(g_{a}\right)^{n} \rightrightarrows_{K} c \text {; } \\
& \text { ii) } C\left(f, \bigcup_{n=0}^{\infty} g_{a}^{n}(K), e^{i \theta}\right)=\{c\} \text {. }
\end{aligned}
$$

Proof of main lemma 1. Let $c \in \mathbb{C}$.

i) $\Rightarrow$ ii). From i) we have

$$
(\forall \varepsilon>0)\left(\exists N_{1}=N_{1}(\varepsilon)\right)\left(\forall n \geq N_{1}\right)(\forall z \in K)\left(\left|f \circ g_{a}^{n}(z)-c\right|<\varepsilon\right)
$$


i.e., $\quad f\left(\bigcup_{n=N_{1}}^{\infty} g_{a}^{n}(K)\right) \subset\{w \in \mathbb{C}|| w-c \mid<\varepsilon\}$.

From lemma 8 we have

$$
\begin{aligned}
& \qquad(\forall \delta>0)\left(\exists N_{2}=N_{2}(\delta)\right)\left(\forall n \geq N_{2}\right)(\forall z \in K)\left(\left|g_{a}^{n}(z)-e^{i \theta}\right|<\delta\right), \\
& \text { i.e., } \quad\left(\forall z \in \bigcup_{n=N_{1}}^{\infty} g_{a}^{n}(K)\right)\left|z-e^{i \theta}\right|<\delta .
\end{aligned}
$$

Let $\left(z_{n}\right)$ be any sequence in $\bigcup_{n=1}^{\infty} g_{a}^{n}(K)$ for which $\lim _{n \rightarrow \infty} z_{n}=e^{i \theta}$. From (5), i.e., from (5') we obtain

$$
\left(\exists N_{3}=N_{3}\left(\left(z_{n}\right), N_{2}\right)\right)\left(\forall n \geq N_{3}\right)\left(z_{n} \in \bigcup_{k=N_{2}}^{\infty} g_{a}^{k}(K)\right) .
$$

If $N_{2} \leq N_{1}$, then we have $\bigcup_{n=N_{2}}^{\infty} g_{a}^{n}(K) \subset \bigcup_{n=N_{1}}^{\infty} g_{a}^{n}(K)$, from this and from (6) it follows that $z_{n} \in \bigcup_{n=N_{1}}^{\infty} g_{a}^{n}(K)$ for every $n \geq N_{3}$. Having in mind now (4) it follows that $\left(\forall n \geq N_{3}\right)\left(\left|f\left(z_{n}\right)-c\right|<\varepsilon\right)$, which means that $\lim _{n \rightarrow \infty} f\left(z_{n}\right)=c$.

If $N_{1} \leq N_{2}$, then from the sequence $\left(z_{n}\right)$, except $z_{1}, \ldots, z_{N_{3}}$, remove those that are in the set $\bigcup_{k=1}^{N_{1}} g_{a}^{k}(K)$, there are only finite many of them. Therefore, there exists $N_{4}$ such that $z_{n} \in \bigcup_{n=N_{1}}^{\infty} g_{a}^{n}(K)$ for every $n \geq N_{4}$. Now, according to (4) we obtain $\left(\forall n \geq N_{3}\right)\left(\left|f\left(z_{n}\right)-c\right|<\varepsilon\right)$, i.e., in this case we also have $\lim _{n \rightarrow \infty} f\left(z_{n}\right)=c$.

Therefore, for every sequence $\left(z_{n}\right)$ in $\bigcup_{n=0}^{\infty} g_{a}^{n}(K)$ we have $\lim _{n \rightarrow \infty} f\left(z_{n}\right)=c$, do we may conclude that $C\left(f, \bigcup_{n=0}^{\infty} g_{a}^{n}(K), e^{i \theta}\right)=\{c\}$.

ii ) $\Rightarrow$ i). From ii) we have $\forall\left(z_{n}\right) \subset \bigcup_{n=0}^{\infty} g_{a}^{n}(K) \wedge \lim _{n \rightarrow \infty} z_{n}=e^{i \theta} \Rightarrow \lim _{n \rightarrow \infty} f\left(z_{n}\right)=c$ i.e.,

$$
(\forall \varepsilon>0)(\exists \delta=\delta(\varepsilon))\left(\forall z \in \bigcup_{n=0}^{\infty} g_{a}^{n}(K)\right)\left(\left|z-e^{i \theta}\right|<\delta \Rightarrow|f(z)-c|<\varepsilon\right)
$$

Since $\mathrm{g}_{a}{ }^{\mathrm{n}} \exists_{\mathrm{K}} \mathrm{e}^{\mathrm{i} \theta}$, for $\delta>0$ the exists $N=N(\delta)$ such that for any $n \geq N$ and every $z \in K$ holds $\left|g_{a}^{n}(z)-e^{i \theta}\right|<\delta$, i.e., 


$$
\bigcup_{n=N}^{\infty} g_{a}^{n}(K) \subset\left\{z \in D|| z-e^{i \theta} \mid<\delta\right\} .
$$

From (7) and (8) it follows that $f\left(\bigcup_{n=N_{1}}^{\infty} g_{a}^{n}(K)\right) \subset\{w \in \mathbb{C}|| w-c \mid<\varepsilon\}$, and therefore $(\forall n \geq N)(\forall z \in K)\left(\left|f\left(g_{a}^{n}(z)\right)-c\right|<\varepsilon\right)$, i.e., $\quad f_{\circ}\left(g_{a}\right)^{n} \rightrightarrows_{K} c$.

If $c=\infty \in \overline{\mathbb{C}}$, the proof goes in the same way as in the case $c \in \mathbb{C}$ instead of the Eucilean metric we have to take the spherical distance.

Main lemma 2. For any function $f: D \rightarrow \overline{\mathbb{C}}$, and a compact set $K, K \subset D$, and any mapping $g_{u} \in P_{D}^{\theta}, g_{u} \neq i$, the following conditions are equivalent:

i) $\quad f o(g u)^{n} \rightrightarrows_{K} c, c \in \mathbb{C}$.

ii) $C\left(f, \bigcup_{n=0}^{\infty} g_{u}^{n}(K), e^{i \theta}\right)=\{c\}$.

Main Lemma 2 may be proved in the same way as Main Lemma 1.

\section{APPLICATIONS}

For $g_{a} \in H_{D}^{\theta}, \quad g_{a} \neq i, \quad$ for which $e^{i \theta}$ is an attraction fixed point $H_{g_{a}}^{\theta}=\left\{g_{a}^{n} \mid n \in \mathbb{N} \cup\{0\}\right\}, a \in(-1,1)$ is fixed, is the hyperbolic semigroup of $\mathrm{G}$ with fixed attraction point $e^{i \theta}$, and $P_{g_{u}}^{\theta}=\left\{g_{u}^{n} \mid n \in \mathbb{N} \cup\{0\}\right\}, u \in(-\infty,+\infty)$ is fixed, is the parabolic semigroup of $\mathrm{G}$ with attraction fixed point $e^{i \theta}$.

\subsection{Angular boundary values of meromorphic functions}

Theorem 1. Let $f: D \rightarrow \overline{\mathbb{C}}$ be a meromorphic function. If

$$
\left\{f \circ g \mid g \in H_{g_{a}}^{\theta}\right\}=\left\{f \circ g_{a}^{n} \mid n \in \mathbb{N} \cup\{0\}\right\}, a \in(-1,1)
$$

(a is fixed), is normal family of functions on the disk $D, \gamma$ is simple Jordan curve with one endpoint in $e^{i \theta}$ and $\gamma \subset \tilde{\Delta}_{H}(\theta, r)$ and $C\left(f, \gamma, e^{i \theta}\right)=\{c\}, c \in \overline{\mathbb{C}}$, then

$$
C\left(f, \Delta\left(e^{i \theta}, \alpha\right), e^{i \theta}\right)=\{c\} \text { for every } \alpha, 0<\alpha<\frac{\pi}{2},
$$

i.e., $e^{i \theta}$ is the Fatou point of the function $f$. 
Proof of Theorem 1. Since $\left\{f \circ g_{a}^{n} \mid n \in \mathbb{N} \cup\{0\}\right\}, a \in(-1,1)$, $a$ is fixed, is a normal family of functions on the disk $\mathrm{D}$, there exists a sequence $\left(f \circ g_{a}^{n_{k}}\right)$ which uniformly on compact sete convegre to a meromorphic function $\varphi$ on $\overline{D_{r_{1}}}$. i.e., $f o\left(g_{a}\right)^{n_{k}} \rightrightarrows \overline{D_{r_{1}}} \varphi$.

Since $\gamma \subset \tilde{\Delta}_{H}(\theta, r)$, the sets $\gamma \cap g_{a}^{n_{k}}\left(D_{r_{1}} \backslash D_{r}\right), 0<r<r_{1}<1, n \in \mathbb{N}$, are made of two simple curves. By $\gamma_{k}$ we denote one of them Then we have $\gamma_{k} \cap \gamma_{k+1}=\varnothing$, and $\left[\Gamma_{k}=g_{a}^{-n_{k}}\left(\gamma_{k}\right)\right] \cap\left[\Gamma_{k+1}=g_{a}^{-n_{k+1}}\left(\gamma_{k+1}\right)\right]=\varnothing, n \in \mathbb{N}$, since the Moebius transforms $g_{a}^{n}$ are bijections

For every $m \in \mathrm{N}$ let us select a sequence $\left(z_{k}^{m}\right), z_{k}^{m} \in \Gamma_{k}$, such that $\lim _{k \rightarrow \infty} z_{k}^{m}=z_{0}^{m} \in \overline{D_{p h}}\left(0, r_{1}\right)$ and $z_{0}^{i} \neq z_{0}^{j}$ for $i \neq j$. We will show that $\varphi\left(z_{0}^{m}\right)=c, c \in \bar{C}$, for every $m \in \mathrm{N}$.

For every $m \in \mathrm{N}$ there holds

$d_{S}\left(\varphi\left(z_{0}^{m}\right), c\right) \leq d_{S}\left(\varphi\left(z_{0}^{m}\right), \varphi\left(z_{k}^{m}\right)\right)+d_{S}\left(\varphi\left(z_{k}^{m}\right), f_{n_{k}}\left(z_{k}^{m}\right)\right)+d_{S}\left(f_{n_{k}}\left(z_{k}^{m}\right), c\right)$

Let $\varepsilon$ be any positive real number. Since of coninuily of $\varphi$ we have $d_{S}\left(\varphi\left(z_{0}^{m}\right), \varphi\left(z_{k}^{m}\right)\right)<\frac{\varepsilon}{3}$, if $\mathrm{k}$ is enough big.

Since the sequence $\left(f_{n_{k}}\right)$ converge uniformly on compact sets of the disk $D$ to $\varphi$, we have $d_{S}\left(\varphi(z), f_{n_{k}}(z)\right)<\frac{\varepsilon}{3}$ for every $z \in \overline{D_{r_{1}}}$ and enough big k.

Since $z_{k}^{m} \in \overline{D_{r}} \quad$ we have $d_{s}\left(\varphi\left(z_{k}^{m}\right), f_{n_{k}}\left(z_{k}^{m}\right)\right)<\frac{\varepsilon}{3}$. Since $\quad z_{k}^{m} \in \Gamma_{k}$ it follows that $\varphi_{n_{k}}\left(z_{k}^{m}\right)=w_{k}^{m} \in \gamma_{k} \subset \gamma$ and $\lim _{k \rightarrow \infty} w_{k}^{m}=e^{i \theta}$.

Since $c$ is the asymptotic value of $f$ snd since $\lim _{k \rightarrow \infty} f_{n_{k}}\left(w_{k}^{m}\right)=\lim _{k \rightarrow \infty} f \circ \varphi_{n_{k}}\left(z_{k}^{m}\right)=\lim _{k \rightarrow \infty} f_{n_{k}}\left(z_{k}^{m}\right)=c$, for enough big k we have $d_{S}\left(f_{n_{k}}\left(z_{k}^{m}\right), c\right)<\frac{\varepsilon}{3}$ for every $m \in \mathrm{N}$.

From (9) and obtained inequality it follows that $d_{s}\left(\varphi\left(z_{0}^{m}\right), c\right)<\varepsilon$ for every $m$. Since $\varepsilon$ is any number, we have $\varphi\left(z_{0}^{m}\right)=c$ for every $m$.

Since the sequence $\left(z_{0}^{m}\right)$ is in $\overline{D_{r_{1}}}$ and i $\overline{D_{r_{1}}}$ has an accumulation point, from the uniqness theorem we have $\varphi \equiv c$.

Therefore, we have proved that any sequence in the family $\left\{f \circ g_{a}^{n} \mid n \in \mathbb{N} \cup\{0\}\right\}$ which is uniformly convergent on compact sets in $D$, is convergent to the constant c. 
Now we will show that any sequence in the family $\left\{f \circ g_{a}^{n} \mid n \in \mathbb{N} \cup\{0\}\right\}$ converge uniformly on compact sets of $\mathrm{D}$ the the constant c. Assume conctrary, that thereexist a sequence postoji $\left(f_{n}\right)$, $f_{n} \in\left\{f \circ g_{a}^{n} \mid n \in \mathbb{N} \cup\{0\}\right\}$, which uniformly on compacts does not converge to the constant c. Then there exists a number $\varepsilon>0$ such that for every $k \in \mathrm{N}$ we have $n_{k} \in \mathrm{N}$ and $z_{n_{k}} \in \overline{D_{r}}$ such that $d_{S}\left(f_{n_{k}}\left(z_{n_{k}}\right), c\right) \geq \varepsilon$. Since tha family $\left\{f \circ g_{a}^{n} \mid n \in \mathbb{N} \cup\{0\}\right\}$ is normal, $f_{n_{k}}$ has a subsequence $f_{n_{k l}}$ which uniformly on compact sets of $D$ converge, according to the preceding consideration it follows that it converge to the constant c, which is contrary with the assumption $d_{S}\left(f_{n_{k}}\left(z_{k}^{m}\right), c\right) \geq \varepsilon$. This contradiction shows that every sequence in $\left\{f \circ g_{a}^{n} \mid n \in \mathbb{N} \cup\{0\}\right\}$ uniformly on compact sets of $D$ converge to the constant c. Having in mind the Lemma 1 it follows that $C\left(f, \bigcup_{n=0}^{\infty} g_{a}^{n}(K), e^{i \theta}\right)=\{c\}$, for every compact set $\mathrm{K}, K \subset D$, and form Lemma 5 and Lemma 18 we have that $C\left(f, \Delta(\theta, \alpha), e^{i \theta}\right)=\{c\}$ for every $\alpha,-\frac{\pi}{2}<\alpha<\frac{\pi}{2}$, i.e., the function $f$ in the point $e^{i \theta}$ has angular boundary value $c$.

In [27] it is proved that $\left\{f \circ g_{a}^{n} \mid n \in \mathbb{N} \cup\{0\}\right\}, a \in(-1,1)$ is normal family on $D$ if and only if in the domain $\tilde{\Delta}_{H}(\theta, r), r \in(0,+\infty)$, does not exist P-sequences for $f$.

Theorem 2. Let $f: D \rightarrow \overline{\mathbb{C}}$ be a meromorphic function. If $\left\{f \circ g_{a}^{n} \mid n \in \mathbb{N} \cup\{0\}\right\}, a \in(-1,1)$, where $a$ is fixed, normal family of functions on the disk $D$ and if for a sequence $\left(z_{n}\right) \subset \tilde{\Delta}_{H}(\theta, r)$ holds $\lim _{n \rightarrow \infty} z_{n}=e^{i \theta}$ and $\lim _{n \rightarrow \infty} f\left(z_{n}\right)=c, c \in \overline{\mathbb{C}}$, then for any sequence $\left(r_{n}\right)$ for which $\lim _{n \rightarrow \infty} r_{n}=0$ and $\bigcup_{n=1}^{\infty} D_{h}\left(z_{n}, r_{n}\right) \subset \tilde{\Delta}_{H}\left(\theta, r_{1}\right)$ for $r_{1}>0, C\left(f, \bigcup_{n=1}^{\infty} D_{h}\left(z_{n}, r_{n}\right), e^{i \theta}\right)=\{c\}$.

Theorem 2 follows directly from theorem on P-sequences which is formulated in the Section 4 and the criteria for normality formulated above of the familiy of functions $\left\{f \circ g_{a}^{n} \mid n \in \mathbb{N} \cup\{0\}\right\}, a \in(-1,1)$ on $D$.

Theorem 3. Let $f: D \rightarrow \overline{\mathbb{C}}$ be meromorphic function. If $\left\{f \circ g_{a}^{n} \mid n \in \mathbb{N} \cup\{0\}\right\}, a \in(-1,1)$ where $a$ is fixed, is normal family of functions on the disk $D$ and if for a sequence $\left(z_{n}\right) \subset \tilde{\Delta}_{H}(\theta, r)$ holds: $\quad \lim _{n \rightarrow \infty} z_{n}=e^{i \theta}, \quad \lim _{n \rightarrow \infty} d_{h}\left(z_{n}, z_{n+1}\right)=0$ and $\lim _{n \rightarrow \infty} f\left(z_{n}\right)=c, c \in \overline{\mathbb{C}}$, then $C\left(f, \Delta\left(e^{i \theta}, \alpha\right), e^{i \theta}\right)=\{c\}$ for every $\alpha, 0<\alpha<\frac{\pi}{2}$, i.e., $e^{i \theta}$ is the Fatou point of the function $f$. 
Proof of Theorem 3. Let $x_{n}=2 d_{h}\left(z_{n}, z_{n+1}\right)$. Then we have $\lim _{n \rightarrow \infty} x_{n}=0$. If $D_{h}\left(z_{n}, x_{n}\right)=\left\{z \mid d_{h}\left(z, z_{n}\right)<x_{n}\right\}$, then form Theorem 2 follows that $C\left(f, \bigcup_{n=1}^{\infty} D_{h}\left(z_{n}, x_{n}\right), e^{i \theta}\right)=\{c\} c \in \overline{\mathbb{C}}$. Since the curve (poligonal line) $\gamma=\overline{z_{1} z_{2} \ldots z_{n} \ldots e^{i \theta}} \subset \bigcup_{n=1}^{\infty} D_{h}\left(z_{n}, x_{n}\right)$, we have $C\left(f, \gamma, e^{i \theta}\right)=\{c\}$. Since it is possible to chose $r>0$, such that $\bigcup_{n=1}^{\infty} D_{h}\left(z_{n}, x_{n}\right) \subset \tilde{\Delta}_{H}(\theta, r)$, from Theorem 1 we conclude $C\left(f, \Delta\left(e^{i \theta}, \alpha\right), e^{i \theta}\right)=\{c\}$ for every $\alpha, 0<\alpha<\frac{\pi}{2}$, i.e., $e^{i \theta}$ is the Fatou point of $f$.

Theorem 4. Let $f: D \rightarrow \overline{\mathbb{C}}$ be meromorphic function such that $f(z) \neq c, c \in \overline{\mathbb{C}}, z \in D$. If $\left\{f \circ g_{a}^{n} \mid n \in \mathbb{N} \cup\{0\}\right\}, a \in(-1,1)$, a is fixed, normal family $f$ functions on th disk $D$ and if holds: $\lim _{n \rightarrow \infty} z_{n}=e^{i \theta}$ and $\lim _{n \rightarrow \infty} f\left(g_{a}^{n}(0)\right)=c, c \in \overline{\mathbb{C}}$, then we have $C\left(f, \Delta\left(e^{i \theta}, \alpha\right), e^{i \theta}\right)=\{c\}$ for every $\alpha, 0<\alpha<\frac{\pi}{2}$, i.e., $e^{i \theta}$ is the Fatou point of $f$.

Proof of Theorem 4. Form normality of meromorphic functions $\left\{f \circ g_{a}^{n} \mid n \in \mathbb{N} \cup\{0\}\right\}$ and the condition $\lim _{n \rightarrow \infty} f\left(g_{a}^{n}(0)\right)=c, c \in \overline{\mathbb{C}}$, from Hurwitz theorem (see [20]) fog $_{\mathrm{u}}{ }^{\mathrm{n}} \rightrightarrows_{\mathrm{K}} \mathrm{c}$ for every compact set $K \subset D$. If we take $K=\overline{D_{h}(0, r)}, 0<\mathrm{r}<1$, then from the Main Lemma 1, Lemma 5 and Lemma 18 we have $C\left(f, \Delta\left(e^{i \theta}, \alpha\right), e^{i \theta}\right)=\{c\}$ for every $\alpha, 0<\alpha<\frac{\pi}{2}$, i.e., $e^{i \theta}$ is the Fatou point of $\mathrm{f}$.

\subsection{Tangentialy oricyclic boundary values of meromorphic functions}

If for every $r \in(0,+\infty)$ holds $C\left(f, \tilde{\Delta}_{o}(\theta, r), e^{i \theta}\right)=\{\omega\}, \omega \in \overline{\mathbb{C}}$, then we will call $\omega$ the upper oricyclic boundary value of $\mathrm{f}$ in the point $e^{i \theta}$. On the other hand, if for every $r \in(0,+\infty)$ holds $C\left(f, \tilde{\tilde{\Delta}}_{o}(\theta, r), e^{i \theta}\right)=\{\omega\}, \omega \in \overline{\mathbb{C}}, \omega \in \overline{\mathbb{C}}$, then we will call $\omega$ the lower o oricyclic boundary value for $f$ in the point $e^{i \theta}$. If $C\left(f, \tilde{\Delta}_{o}(\theta, r) \cup \tilde{\Delta}_{o}(\theta, r), e^{i \theta}\right)=\{\omega\}$ then we call se $\omega$ the oricyclic boundary value of $\mathrm{f}$ in the point $e^{i \theta}$.

Theorem 5. Let $f: D \rightarrow \overline{\mathbb{C}}$ be meromorphic function. If 


$$
\left\{f \circ g \mid g \in P_{g_{u}}^{\theta}\right\}=\left\{f \circ g_{u}^{n} \mid n \in \mathbb{N} \cup\{0\}\right\}, u \in(0, \infty),
$$

$u$ is fixed, normal family of functions on the disk $D$ and if for a sequence $\left(z_{n}\right) \subset \tilde{\Delta}_{o}(\theta, r)$ holds $\lim _{n \rightarrow \infty} z_{n}=e^{i \theta}$ and $\lim _{n \rightarrow \infty} f\left(z_{n}\right)=c, c \in \overline{\mathbb{C}}$, then for every sequence $\left(r_{n}\right)$ for which $\lim _{n \rightarrow \infty} r_{n}=0$ and $\bigcup_{n=1}^{\infty} D_{h}\left(z_{n}, r_{n}\right) \subset \tilde{\Delta}_{o}\left(\theta, r_{1}\right)$ for a $r_{1}>0, C\left(f, \bigcup_{n=1}^{\infty} D_{h}\left(z_{n}, r_{n}\right), e^{i \theta}\right)=\{c\}$.

Theorem 5'. Let $f: D \rightarrow \overline{\mathbb{C}}$ be a meromorphic function. If

$$
\left\{f \circ g \mid g \in P_{g_{u}}^{\theta}\right\}=\left\{f \circ g_{u}^{n} \mid n \in \mathbb{N} \cup\{0\}\right\}, u \in(-\infty, 0),
$$

where $u$ is fixed, normal family of functions on the disk $D$ and if for a sequence $\left(z_{n}\right) \subset \tilde{\tilde{\Delta}}_{o}(\theta, r)$ holds: $\lim _{n \rightarrow \infty} z_{n}=e^{i \theta}$ and $\lim _{n \rightarrow \infty} f\left(z_{n}\right)=c, c \in \overline{\mathbb{C}}$, then for every $\left(r_{n}\right)$ for which $\lim _{n \rightarrow \infty} r_{n}=0$ and $\bigcup_{n=1}^{\infty} D_{h}\left(z_{n}, r_{n}\right) \subset \tilde{\Delta}_{o}(\theta, r)$ for $r_{1}>0, C\left(f, \bigcup_{n=1}^{\infty} D_{h}\left(z_{n}, r_{n}\right), e^{i \theta}\right)=\{c\}$.

U [27] it is proved that $\left\{f \circ g_{u}^{n} \mid n \in \mathbb{N} \cup\{0\}\right\}, u \in(-\infty, 0)$ is normal family on the disk $D$ if and only if in the domain $\tilde{\tilde{\Delta}}_{o}(\theta, r) \tilde{\Delta}_{o}(\theta, r), r \in(0,+\infty)$, does not exist P-sequences for the function $f$. On the other hand, the family $\left\{f \circ g_{u}^{n} \mid n \in \mathbb{N} \cup\{0\}\right\}, u \in(0,+\infty)$ is normal on the disk $D$ if and only if in the domain $\tilde{\Delta}_{o}(\theta, r), r \in(0,+\infty)$, does not exist $P$-sequences for the function $\mathrm{f}$.

Theorem 5 and Thorem $5^{\prime}$ follows directly from theorem on $P$-sequences 1 which is formulated in Section 4 and the above formulated criterion for normality of the family of functions $\left\{f \circ g_{u}^{n} \mid n \in \mathbb{N} \cup\{0\}\right\}, u \in(-\infty, 0)\left\{f \circ g \mid g \in P_{g_{u}}^{\theta}\right\}=\left\{f \circ g_{u}^{n} \mid n \in \mathbb{N} \cup\{0\}\right\}, u \in(-\infty, 0)$.

Theorem 6. Let $f: D \rightarrow \overline{\mathbb{C}}$ be a meromorphic function. If

$$
\left\{f \circ g \mid g \in P_{g_{u}}^{\theta}\right\}=\left\{f \circ g_{u}^{n} \mid n \in \mathbb{N} \cup\{0\}\right\}, u \in(0, \infty), u \text { is fixed, }
$$

is normal family on the disk $D, \gamma$ a simple Jordan curve with one endpoint in $e^{i \theta}$ and $\gamma \subset \tilde{\Delta}_{o}(\theta, r)$ and $C\left(f, \gamma, e^{i \theta}\right)=\{\omega\}, \omega \in \mathbb{C}$, then $C\left(f, \tilde{\Delta}_{o}(\theta, r), e^{i \theta}\right)=\{\omega\}$ for every $r \in(0,+\infty)$, i.e., $\omega$ is the upper oricyclic boundary value for the function $f$ in the point $e^{i \theta}$. 
Theorem 6'. Let $f: D \rightarrow \overline{\mathbb{C}}$ be a meromorphic function. If

$$
\left\{f \circ g \mid g \in P_{g_{u}}^{\theta}\right\}=\left\{f \circ g_{u}^{n} \mid n \in \mathbb{N} \cup\{0\}\right\}, u \in(-\infty, 0), u \text { is fixed, }
$$

is normal family of functions on the disku $D, \gamma$ is simple Jordan curve with one endpoint in $e^{i \theta}$ and $\gamma \subset \tilde{\tilde{\Delta}}_{o}(\theta, r)$ and $C\left(f, \gamma, e^{i \theta}\right)=\{\omega\}, \omega \in \mathbb{C}$, then $C\left(f, \tilde{\tilde{\Delta}}_{o}(\theta, r), e^{i \theta}\right)=\{\omega\}$ for every $r \in(0,+\infty)$, i.e., $\omega$ is the lower oricyclic boundary value of the function $f$ in the point $e^{i \theta}$.

Theorem 6 and Theorem 6' may be proved using the Main Lemma 2 and Lemma 19 in the same way as Theorema 1 is derived from the Main Lemma 1 and Lemma 18.

Theorem 7 and Theorem $7^{\prime}$ may be proved using Theorem 6 and Theorem $6^{\prime}$ in the same way as Theorem 3 using Theorem 1.

Theorem 7. Let $f: D \rightarrow \overline{\mathbb{C}}$ be a meromorphic function. If

$$
\left\{f \circ g \mid g \in P_{g_{u}}^{\theta}\right\}=\left\{f \circ g_{u}^{n} \mid n \in \mathbb{N} \cup\{0\}\right\}, u \in(0,+\infty), u \text { is fixed, }
$$

is a normal family of functions on the disk $D$ and if for a sequence $\left(z_{n}\right) \subset \tilde{\Delta}_{o}(\theta, r)$ holds: $\lim _{n \rightarrow \infty} z_{n}=e^{i \theta}, \quad \lim _{n \rightarrow \infty} d_{h}\left(z_{n}, z_{n+1}\right)=0$ and $\lim _{n \rightarrow \infty} f\left(z_{n}\right)=c, c \in \overline{\mathbb{C}}$, then $C\left(f, \tilde{\Delta}_{o}(\theta, r), e^{i \theta}\right)=\{\omega\}$ for every $r \in(0,+\infty)$, i.e., $\omega$ is the upper oricyclic boundary value of function $f$ in the point $e^{i \theta}$.

Theorem 7!. Let $f: D \rightarrow \overline{\mathbb{C}}$ be a meromorphic function. If

$$
\left\{f \circ g \mid g \in P_{g_{u}}^{\theta}\right\}=\left\{f \circ g_{u}^{n} \mid n \in \mathbb{N} \cup\{0\}\right\}, u \in(-\infty, 0), u \text { is fixed, }
$$

is normal family of functions on the disk $D$ and if for a sequence $\left(z_{n}\right) \subset \tilde{\tilde{\Delta}}_{o}(\theta, r)$ holds: $\lim _{n \rightarrow \infty} z_{n}=e^{i \theta}, \quad \lim _{n \rightarrow \infty} d_{h}\left(z_{n}, z_{n+1}\right)=0$ and $\lim _{n \rightarrow \infty} f\left(z_{n}\right)=c, c \in \overline{\mathbb{C}}$, then $C\left(f, \tilde{\tilde{\Delta}}_{o}(\theta, r), e^{i \theta}\right)=\{\omega\}$ for every $r \in(0,+\infty)$, i.e., $\omega$ is the lower oricyclic boundary value of the function $f$ in the point $e^{i \theta}$.

Theorem 8. Let $f: D \rightarrow \overline{\mathbb{C}}$ be a meromorphic function such that $f(z) \neq c, c \in \overline{\mathbb{C}}, z \in D$. $\left\{f \circ g \mid g \in P_{g_{u}}^{\theta}\right\}=\left\{f \circ g_{u}^{n} \mid n \in \mathbb{N} \cup\{0\}\right\}, u \in(0,+\infty), u$ is fixed, normal family of functions on $D$ and if $\lim _{n \rightarrow \infty} z_{n}=e^{i \theta}$ and $\lim _{n \rightarrow \infty} f\left(g_{u}^{n}(0)\right)=c, c \in \overline{\mathbb{C}}$, then we have $C\left(f, \tilde{\Delta}_{o}(\theta, r), e^{i \theta}\right)=\{\omega\}$ for every $r \in(0,+\infty)$, i.e., $\omega$ is the upper oricyclic boundary value of the function $f$ in the point $e^{i \theta}$. 
Theorem 8!. Let $f: D \rightarrow \overline{\mathbb{C}}$ be a meromorphic function such that $f(z) \neq c, c \in \overline{\mathbb{C}}, z \in D$. $\left\{f \circ g \mid g \in P_{g_{u}}^{\theta}\right\}=\left\{f \circ g_{u}^{n} \mid n \in \mathbb{N} \cup\{0\}\right\}, u \in(-\infty, 0), u$ is fixed, normal family of functions on the disk $D$ and if $\lim _{n \rightarrow \infty} z_{n}=e^{i \theta}$ and $\lim _{n \rightarrow \infty} f\left(g_{u}^{n}(0)\right)=c, c \in \overline{\mathbb{C}}$, then $C\left(f, \tilde{\tilde{\Delta}}_{o}(\theta, r), e^{i \theta}\right)=\{\omega\}$ for every $r \in(-\infty, 0)$, i.e.. $\omega$ is the lower oricyclic boundary value of the function $f$ in $e^{i \theta}$.

Theorem 8 and Theorem 8' may be proved using the Main Lemma 2 and Lemma 19 in the same way as Theorem 4 is derived from the Main Lemma 1, Lemma 5 and Lemma 18.

\section{CONSTRUCTION OF ONE EXAMPLE}

We will construct an example of meromorphic function $f: D \rightarrow \overline{\mathbb{C}}$ for which $\left\{f \circ g_{a}^{n} \mid n \in \mathbb{N} \cup\{0\}\right\}, \quad a \in(-1,1)$, a is fixed, is normal family of functions on the disk $D$, and $\left\{f \circ g_{a}^{\theta} \mid g_{a}^{\theta} \in H_{D}^{\theta}\right\}$ is not normal family of functions on $D$. This construction is similar as one in the work [8].

Let $z_{k}=-\rho_{k} e^{i \theta}, \rho_{k}>0, k \in \mathbb{N}$, be such that $\lim _{k \rightarrow \infty} \rho_{k}=1$ and $\lim _{k \rightarrow \infty} d\left(z_{k}, z_{k+1}\right)=0$. The elements of the sequence $\left(z_{k}\right)$ are in the set $P_{\theta} \cap \Delta_{g^{-1}}(-\theta, r)$. Let a sequence $\left(\varepsilon_{k}\right)$ be a such one that we have:

$0<\varepsilon_{k+1}<\varepsilon_{k} ; \quad \lim _{k \rightarrow \infty} \varepsilon_{k}=0 ; \quad D\left(z_{k}, \varepsilon_{k}\right) \cap D\left(z_{k+1}, \varepsilon_{k+1}\right)=\varnothing, k \in \mathbb{N} ; \quad \lim _{k \rightarrow \infty}\left(\sup _{z \in D\left(z_{k}, \varepsilon_{k}\right)} d\left(z_{k}, z_{k+1}\right)\right)=0 ;$ $\sum_{k=1}^{\infty} \varepsilon_{k}<+\infty$

Let $a_{k}=\varepsilon_{k}^{3}, k \in \mathbb{N}$, and $f(z)=\sum_{k=1}^{\infty} a_{k}\left(z-z_{k}\right)^{-1}$. The function $\mathrm{f}$ is meromorphic on the disk $\mathrm{D}$, with the poles in $z_{k}, k \in \mathbb{N}$. Since $f\left(z_{k}\right)=\infty,\left|f\left(z_{k}+\varepsilon\right)\right|<M, k \in \mathbb{N}$, and $\lim _{n \rightarrow \infty} d_{h}\left(z_{k}, z_{k}+\varepsilon\right)=0$

from Theorem 2 on $P$-sequences it follows that $\left(z_{k}\right) \subset \Delta^{g}{ }_{-1}(-\theta, r) \subset \Delta(\theta, r)$ is $P$-sequence of $f$. Therefore, we may conclude that $\left\{f \circ g_{a}^{\theta} \mid g_{a}^{\theta} \in H_{D}^{\theta}\right\}$ is not normal family of functions on the disk D.

Since for every $z^{\prime}, z^{\prime \prime} \in D \backslash \bigcup_{k=1}^{\infty} D\left(z_{k}, \varepsilon_{k}\right)$ we have $\left|f\left(z^{\prime}\right)-f\left(z^{\prime \prime}\right)\right| \leq\left|z_{k}{ }^{\prime}-z_{k}^{\prime \prime}\right| \sum_{k=1}^{\infty} \varepsilon_{k}=C<+\infty$ and since $\Delta_{g}(\theta, r), r>0$, contains finite number of points $z_{k}$, and since $\Delta_{g}(\theta, r), r>0$, is invariant set 
with respect to $g_{a}^{n}, n \in \mathbb{N}$, it follows that $\limsup _{\Delta_{g}(\theta, r)_{\exists z} \rightarrow e^{i \theta}}|f(z)|=c_{f}(r)<\infty, 0<r<1$. Therefore, for every $r, 0<r<1$, the function $f$ is bounded on $O_{r} \cap \Delta_{g}(\theta, r)$, where $O_{r}=\left\{z||\left|z-e^{i \theta}\right|<1-r\right\}$ so we have that $\left\{f \circ g_{a}^{n} \mid n \in \mathbb{N} \cup\{0\}\right\}, a \in(-1,1)$ is normal family on the disku $D$ (see [20], p. 35, Montel's theorem).

\section{CONCLUSION}

In this paper it is given a new approach in deriving theorems from the theory of asymptotical behavior of analytic functions. Namely, our theorems are proved using some results from the dynamic and the geometry of Möebious mappings and classical uniqueness theorem for analytic mappings, but in the preceding time these theorems were proved by using the approach and the results from the theory of harmonic mappings and harmonic measure theory.

The Main Lemma 1 and the Main Lemma 2 prove that the necessary condition for a function $f: D \rightarrow \overline{\mathbb{C}}$ to has the angular or oricyclic boundary value in $e^{i \theta}$ is that the following two families of functions

$$
\left\{f \circ g_{a}^{n} \mid n \in \mathbb{N} \cup\{0\}\right\}, a \in(-1,1),\left\{f \circ g \mid g \in P_{g_{u}}^{\theta}\right\}=\left\{f \circ g_{u}^{n} \mid n \in \mathbb{N} \cup\{0\}\right\}, u \in(-\infty, 0),
$$

are normal on the disk $\mathrm{D}$.

The constructed example in Section 7 shows that the angular boundary values exist for broader class of meromorphic functions then the class considered in the theorems of Lehto-Virtanen and Gavrilov-Burkova. We have proved theorems of type of Bagemihl-Seidel for broader class of functions.

From Theorem 6 and Theorem $6^{\prime}$ it follows that the upper and the lower oricyclic boundary values of meromorphic function $f: D \rightarrow \overline{\mathbb{C}}$ in $e^{i \theta}$ are equal $\omega, \omega \in \overline{\mathbb{C}}$, then $\mathrm{f}$ has tangential oricyclic boundary value $\omega$ in $e^{i \theta}$. In general case it is possible to occur that one of these boundary values exists but the other not. This may be proved by an example which may be constructed in a similar way as the example in the Section 7.

For further consideration it remains to consider is it possible to use the approach of this paper in order to derive results concerning the asymptotic behavior of harmonic functions on the unit disk $D$ in the complex plane $\mathbb{C}$. 
Acknowledgements: This paper is supported by the Science Support Program at the University of Montenegro, the Program for Stimulating Publication in Open Access Magazines in 2018 of the Ministry of Science of Montenegro and the Program Competitiveness of NRNU MEPhI.

\section{REFERENCES}

[1] F. Bagemihl and W. Seidel, "Sequential and continues limits of meromorphic functions", Ann. Acad. Sci. Fenn. Ser. A. I., 280, (1960).

[2] A.F. Beardon, The Geometry of Discrete Groups, Springer (1995).

[3] E.F. Collingwood, A.J. Lohwater, The theory of cluster sets, Cambridge University Press (1966).

[4] P. Gauthier, “A criterion for normalcy“, Nagoya Math. J., 32, 277-282 (1968).

[5] P.M. Gauthier, "Cercles de remplissage and asymptotic behaviour along circuitous path", Can. J. Math., 2, 389-393 (1970).

[6] V.I. Gavrilov, "On the distribution of values of non-normal meromorphic functions in the unit disc", Math. Sb. 109 (67), 408-427 (1965).

[7] V.I. Gavrilov, "Behavior along chords of meromorphic functions in the unit disk", Dokl. Akad. Nauk SSSR, 216, 21-23 (1974).

[8] V.I. Gavrilov, E.F. Burkova, "Onmeromorphic functions generating normal families on subgroups of conformal automorphisms of the unit disc”, Dokl Akad Nauk SSSR, 245, 1293-1296 (1979).

[9] G.M. Goluzin, Geometric theory of functions of complex variables, Leningrad, Gos. ed. tehn.theor. Lit., (1952).

[10] B.A. Fuks, Neyevklidova geomeriyav teorii konformnykh i psevdokonformnykh otobrazheniy, Fos. Izd. Tekhniko-teoreticheskoy literatury, Moskva-Leningrad (1951).

[11] O. Lehto and K.I. Virtanen, "Boundary behaviour and normal meropmorfhic functions", Acta Math., 97, 47-65 (1957).

[12] E. Lindelöf, "Sur un principe general de l'analyse et ses applications a la theorie de la representation conforme”, Ada Soc.Sci. Fenn. 46 (4), 1-35 (1915).

[13] A. Lohwater, "The boundary behavior of analytic function”, Itogi Nauki Tehn., Ser. Mat. Anal. 10, 99-259 (1973).

[14] G. D. Lovshina, "On meromorphic functions normal and hypernormal with respect to a Subgroup of the unit disk”, Dokl Akad Nauk SSSR, 252, 438-440 (1980).

[15] J. Milnor, Dynamics in One Complex Variable, Princeton University Press, Princeton And Oxford, (2006).

[16] P. Montel, Lecons sur les familles normals de fonctions analitques et leurs aplications, GauthierVillars, Paris (1927).

[17] Ž. Pavićević, J. Šušić, "Primenemiye tsiklicheskikh svoyst dinamicheskikh sistem k izucheniyu granichnykh predelov proizvolnykh funktsiy“, Doklady A. N., 387 (1), 16-18 (2002).

[18] Zarko Pavicevic, "On Angular Limits of Normal Meromorphic Functions: A Geometric Aspect”, Journal of Complex Analysis, 2014, ID 216398, 1-6 (2014). DOI: 10.1155/9372.

[19] Žarko Pavićevića and Marijan Marković, "Normality and boundary behaviour of arbitrary and meromorphic functions along simple curves and applications", Complex Variables And Elliptic

Equations, 63 (1), 1-22 (2018). 
[20] J.L. Schiff, Normal Families, Universitext, Springer-Verlag, New York, (1993).

[21] W. Seidel, "On the cluster values of analytic functions", Trans. Amer. Math. Soc. 34, 1-21 (1932).

[22] J. Väisälä, “On normal quasiconformal functions”, Ann. Acad. Sci. Fenn. Ser. A. I., 266, 1- 33 (1959).

[23] G. Valiron, Functions analytiques, Press Univ. De France, Paris (1954).

[24] Kh. E. Mehia, Boundary properties of equimorphic functions, Cand. Thesis, Gus.Univ. M.V. Lomonosov, Moskba, (1981).

[25] A.A. Simushev, Prostranstvennyye normal'nyye kvazimeromorfnyye otobraćeniya, Cand. Thesis, Gus.Univ. M.V, Lomonosov, Moskba, (1986).

[26] J. Šušić, Ž. Pavićević, "The normality of meromorphic functions along an arbitrary curve”, Math. Balkanica, 22, 121-131 (2008).

[27] J. Šušić, Dynamical systems and the boundary properties of a function, Ph.D. thesis, Univ. Montenegro, (2002).

Received November 15, 2020 